vol. 24 - $n^{\circ} 3 \mid 2008$

Numéro ouvert

\title{
Le « combat pour l'intégration » des églises issues du Réveil congolais (RDC)
}

The "Fight for Integration" of the Congolese Revival (DRC) Churches

La "lucha para la integració" de las iglesias nacidas del Renacimiento congolès (RDC)

Sarah Demart

\section{QpenEdition}

Journals

Édition électronique

URL : https://journals.openedition.org/remi/4840

DOI : $10.4000 /$ remi.4840

ISSN : $1777-5418$

Éditeur

Université de Poitiers

Édition imprimée

Date de publication : 1 décembre 2008

Pagination : 147-165

ISBN : 978-2-911627-50-7

ISSN : 0765-0752

Référence électronique

Sarah Demart, "Le " combat pour l'intégration » des églises issues du Réveil congolais (RDC) », Revue européenne des migrations internationales [En ligne], vol. 24 - n³ | 2008, mis en ligne le 01 décembre

2011, consulté le 14 avril 2022. URL : http://journals.openedition.org/remi/4840 ; DOI : https://doi.org/ 10.4000/remi.4840 


\title{
Le « combat pour l'intégration » des églises issues du Réveil congolais (RDC)
}

\author{
Sarah DEMART*
}

Au début des années 1980, de nombreux pays européens ont vu s'implanter sur leurs territoires des églises « africaines ». Un grand nombre d'entre elles ${ }^{1}$ est d'origine congolaise (RDC, ex-Zaïre ${ }^{2}$. Bien qu'une estimation soit difficile à établir en regard du caractère indépendant et mouvant qui caractérise ces collectifs religieux, on peut à l'échelle de l'Europe, estimer au nombre de quelques milliers ces nouvelles églises ; environ 200 en Belgique et plus de 500 en France ${ }^{3}$. Toutefois, une minorité seulement est reconnue, c'est-à-dire affiliée aux réseaux protestants locaux. Cette reconnaissance s'opère de façon différenciée selon les contextes nationaux. Ainsi, en Belgique, l'église doit faire une démarche auprès d'une confession protestante locale via l'EPUB (L'Église Protestante Unie de Belgique) ou via le Synode (Fédéral des Églises évangéliques et protestantes de Belgique). En France, par contre, cette « négociation » a été menée sur un mode collectif, à l'initiative des églises zaïroises qui se sont constituées au début des années 2000 en «Communauté des Églises

* Doctorante en sociologie, Université Toulouse le Mirail, Laboratoire Lisst-Cers UMR 5193 Maison de la Recherche, Université de Toulouse le Mirail 5, Allées Antonio Machado, 1058 Toulouse Cedex 9, France. Université Louvain la Neuve, Place Montesquieu, 1, 1348 Louvain-la-Neuve, Belgique, sarahdemart@hotmail.com

1 Notamment en ce qui concerne les Églises francophones. Pour des populations anglophones, on peut se référer aux travaux de Sandra Fancello sur le pentecôtisme ghanéen (2006), et pour une approche plus générale de cette évolution du protestantisme français à ceux de Sébastien Fath (2005).

2 Lorsque le nationaliste Laurent-Désiré Kabila renverse le pouvoir de Mobutu Sese Seko en 1997, il opère un certain nombre de changements, à commencer par la « dézaïrianisation » du pays. Le Zaïre redevient Congo et plus précisément République démocratique du Congo (RDC). La zaïrianisation ayant été le mouvement culturel, politique et économique initié par Mobutu en 1974 dont l'objectif était de revenir à « l'authenticité » africaine.

3 Par «Églises congolaises », j’entends les Églises impulsées par des congolais. Ces Églises ayant pu, et c'est le plus souvent le cas, accueillir des fidèles d'autres nationalités : africaines, européennes etc., un des objectifs majeurs étant de s'ouvrir, d'évangéliser et de sortir de toute forme de « ghetto », pour reprendre le terme le plus souvent évoqué. 
d'Expression Africaines de France » (CEAF), nonobstant la diversification croissante de leurs assemblées ${ }^{4}$. En devenant communauté membre de la FPF (Fédération Protestante de France), la CEAF a alors obtenu l'habilitation à fournir une reconnaissance juridique à ces nouveaux mouvements religieux ${ }^{5}$.

Ces nouvelles églises composent un paysage religieux contrasté, avec souvent guère plus de dix membres à leur début et plusieurs centaines voire quelques milliers, lorsqu'elles sont «instituées ». Elles rassemblent de façon générale, une majorité de femmes et de jeunes. Les cultes ont lieu dans des hangars, des caves, des grandes maisons, ou encore dans des usines désaffectées qui, réaménagées, permettent d'accueillir de grandes assemblées, sans déranger le voisinage. Ces nouveaux temples se développent de façon autonome, grâce aux offrandes et aux dîmes des fidèles ${ }^{6}$. Si l'on a affaire à des dispositifs élaborés dans et par la migration, il ne faut pas cependant oublier que ces églises sont aussi les marqueurs d'un mouvement missionnaire important : le pentecôtisme. Confession protestante encore minoritaire et marginale en Europe, le pentecôtisme n'en demeure pas moins, l'une des principales transnationalisation religieuse du siècle passé (Corten, 2000). Depuis le début du $\mathrm{XX}^{\mathrm{e}}$ siècle, son expansion à une échelle mondiale n'a cessé de multiplier les foyers d'émergence (réveils) de cette religiosité (Willaime, 1999) au point que sa naissance est encore l'objet de controverses. Néanmoins, deux foyers sont communément admis et par ailleurs significatifs de l'extrême plasticité de cette forme religieuse. Celui de la communauté «blanche » dans l'école biblique de Topeka (Kansas) dirigée par le Pasteur méthodiste Charles F. Parnham proche du Klu Klux Klan créée en 1901 et celui de la communauté «noire » d'Azuza Street Mission dirigée par le Pasteur baptiste William J. Seymour, créée en 1906 à Los Angeles 7 . Dès les années 1910, le pentecôtisme s'introduit en Afrique subsaharienne, puis en Europe, au cours des années 1930. Aujourd'hui, à travers le monde, ce sont cinq cents millions de croyants qui sont rattachés à la confession pentecôtiste/charismatique (Barrett, 2001). En référence aux Actes des apôtres (Livres des Actes, chap. II), celle-ci revendique le Salut par la conversion, la glossolalie ou «parler en langues », les prophéties, la guérison miraculeuse des maladies et le retour prochain de Jésus-Christ.

Bien qu'implanté au Congo au début du XX $\mathrm{XX}^{\mathrm{e}}$ siècle via les Assemblées de Dieu, depuis les années 1970, le pentecôtisme connaît un renouveau spectaculaire au sein de la population congolaise. On peut attribuer l'origine de ce « vent de l'esprit Saint », qui

4 Les jeunes nés en France mais aussi leurs parents dont un certain nombre ont acquis la nationalité française ont modifié la composition des assemblées, qui, si elles sont majoritairement dirigées par des Pasteurs d'origine congolaise, comptent de plus en plus de nationalités différentes parmi les fidèles.

5 Dans ce contexte où les églises se forment en dehors d'une autorité centralisatrice, la frontière distinguant l'institution «Église » des lieux de cultes peut être difficile à établir. D'où le flou dans certaines désignations de ces Églises/églises que j'ai choisi de restituer tel quel.

6 Les offrandes sont dominicales : à chaque culte, mais également « spéciales », lorsqu'il s'agit de soutenir un projet : achat d'un terrain ou d'un bâtiment ou de matériel. La dîme est mensuelle et correspond au dixième du salaire.

7 Celui-ci connaîtra d'ailleurs une grande expansion dans la Bible Belt (Fath, 2004). 
caractérise le Réveil, à la venue successive de nombreux évangélistes internationaux de grand renom : de la fin des années $1960^{\circ}$ au milieu des années 1980. Ils ont mené, parfois avec de grands moyens, des campagnes d'évangélisation et des « croisades » pour appeler les foules à se convertir, à Kinshasa comme à l'intérieur du pays. Dans les années 1970, des groupes de prière ont commencé à se former en marge des Églises historiques, protestantes et catholiques, mettant en cause le bien fondé des séparations religieuses, ainsi que l'ordre des centralités du catholicisme, institué depuis l'époque coloniale comme religion dominante. La découverte d'une relation sans médiation avec un Dieu qui, depuis l'avènement de Jésus, «n'a pas changé », peut véritablement être considéré comme le moteur fédérateur de ces chrétiens de différentes tendances. De ces groupes, où chacun pouvait expérimenter les dons charismatiques par la descente de l'Esprit Saint, sont nées les églises des années 1980. Elles ont à leur tour donné lieu à de nombreuses ramifications, notamment à l'échelle internationale, avec de nombreuses scissions. Dans le déploiement de cette religiosité émotionnelle (Willaime, 1999) qui transforma les pratiques habituelles de prière, le pentecôtisme congolais s'est développé sur un mode hybride (Corten et Mary, 2000). Véritable puissance de libération face aux «blocages » que génèrent les forces sorcières ou occultes, la puissance biblique est donnée comme « la solution » à tous les problèmes (santé, travail, mariage, malédiction etc.), qui ne peuvent relever que de l'action des démons et fétiches. Cette nouvelle vague pentecôtiste est d'une grande diversité et l'on observe une fragmentation de ce mouvement dont les parties sont régulièrement en opposition concernant la liturgie, les pratiques économiques et politiques et enfin le leadership. C'est dans cette dynamique que le Réveil congolais s'est développé à l'intérieur comme à l'extérieur du pays. Aujourd'hui, la seule ville de Kinshasa, compte plus de 6000 Églises de réveil auxquelles l'État a accordé la reconnaissance juridique depuis 2001.

Le Congo Brazzaville ou l'Angola, pays frontaliers de la RDC, sont devenus des terres de missions et d'autres pays plus lointains comme l'Afrique du sud, le Canada, les États-Unis et l'Europe, n'échappent pas à cette « fièvre missionnaire », parfois planifiée mais le plus souvent improvisée et souvent propice à des stratégies migratoires (même si les pasteurs voyageant avec un visa missionnaire sont très peu nombreux). En Europe, les premières Églises se sont implantées en France (1983) et en Belgique (1984) à l'initiative de prédicateurs actifs depuis plusieurs années au Congo et qui dans le cadre de l'évangélisation en Europe ont suivi des formations théologiques (souvent de troisième cycle). Aujourd'hui minoritaires ${ }^{9}$, ces Pasteurs ont été rejoints par toute une génération de Pasteurs autoproclamés (fin 1980-1990), qui revendiquent une formation «essentiellement délivrée par l'Esprit-Saint». Les Assemblées se sont également diversifiées avec les flux migratoires liés à la crise socioéconomique pendant la gouvernance mobutiste (1965-1997), les grands pillages

8 La première « croisade » est organisée en 1968 par T.L. Osborn.

9 Par contre, ils sont en France comme en Belgique à l'origine des processus de fédération des Églises. 
militaires (1991-1992) et depuis 1998, l'agression burundo-ougando-rwandaise ${ }^{10}$. Celle-ci a conduit à d'importants déplacements de population vers la capitale et hors du pays. Selon les sources ${ }^{11}$, on estime aujourd'hui entre 500000 et quatre millions, le nombre de Congolais vivant à l'étranger dont environ 20000 en Belgique et 40000 en France, en comptant les personnes en situation irrégulière ${ }^{12}$. L'Europe est donc une destination parmi tant d'autres de ces mouvements migratoires qui se sont, au fil du temps, diversifiés ; l'élite socioprofessionnelle se dirigeant vers d'autres pays Afrique, tandis que les migrations estudiantines se réorientent plutôt vers le Canada et les ÉtatsUnis, à l'instar des migrations africaines contournant les rapports post-coloniaux qui perdureraient en Europe (Mbembe, 2005).

Le développement des Églises, leurs modes d'organisation, leurs demandes de reconnaissance et leurs stratégies d'investissement de l'espace public diffèrent de façon considérable, selon les contextes européens, et selon le profil du dirigeant, mais l'accent "pro-intégration ${ }^{13} \mathrm{du}$ discours religieux est par contre une constante significative. On assiste ainsi à une recomposition du discours religieux dans une perspective où l'intégration citoyenne des croyants, atteste de la puissance et de la grâce divine et de «l'édification » du croyant.

Dans cet article, je mettrai en perspective le « combat pour l'intégration » à partir de données recueillies entre 2003 et 2008 dans une cinquantaine d'églises situées en France, en Belgique et à Kinshasa. La méthode adoptée est celle d'une anthropologie sociale, alternant des entretiens systématiques (semi-dirigés, non-dirigés et informels) auprès des membres et des responsables religieux avec des périodes d'immersion, brèves et longues, au sein de différentes Assemblées. Ce qui concerne : les espaces-temps du culte, les espaces-temps communautaires [comme les mariages coutumiers ou religieux, les matangas (deuils) etc.], les espaces de décision et de formation (les réunions des équipes dirigeantes, les formations pour les prédicateurs, les cours délivrés dans des instituts bibliques ou de «missiologie ») ainsi que l'espace plus privé, des fidèles et des pasteurs.

Quatre aspects seront développés. Premièrement, celui des ressources que fournit l'identité chrétienne à ces migrants pour se penser en dehors des stigmates associés à l'identité et au statut « d'immigré ». Deuxièmement, la façon dont l'Église

10 Improprement désignée comme « crise congolaise » ou « guerre civile », cette « agression » rwando-ougando-burundaise a entrepris une vaste entreprise de pillages et de tueries, utilisant le viol et la contamination au VIH comme arme de guerre et fait à ce jour, selon les sources entre 4 et 5,4 millions de morts. L'Unicef dans son communiqué de presse du 24 juillet 2006 considère que ce drame est comparable à « un tsunami tous les six mois ».

11 Aucune statistique officielle n'étant établie.

12 Mais sans compter les Congolais qui ont acquis la nationalité du pays d'installation, ni les enfants nés en France ou en Belgique, et ce d'autant que la nationalité congolaise est une et indivisible.

13 Pour Chantal Bordes-Benayoun et Dominique Schnapper (2007), l'intégration ne peut être une donnée en soi mais au contraire une reconstruction a posteriori et surtout une mise en perspective du processus interactif entre la société d'installation (ou de transit) et les migrants, qui participent de sa dynamique interne. 
institutionnalise une attente collective qui a fonction de régulation et de canalisation des émotions, suscitées par les situations d'attente ou de «blocage » des croyants. Enfin, dans les deux dernières parties, je m'intéresserai à des composantes de l'espace religieux qui articulent culture d'origine (réelle ou imaginaire) et culture biblique (entendue comme universelle) et qui sont susceptibles de subvertir des logiques qui assignent ces collectifs à l'altérité. La proposition de cette contribution est de considérer que pour ces collectifs de migrants chrétiens, le contexte migratoire actualise un rapport ancien, que récapitule la notion de «blocage » tout en fournissant les conditions d'une rupture " historique » avec l'ordre des hiérarchies et des centralités autochtones qui les situent en marge.

\section{COMMENCER PAR ROMPRE AVEC LES STIGMATES DE « L'IMMIGRÉ »}

Durant les années 1980, la migration a commencé à s'imposer comme une stratégie de survie économique et de «Salut» pour nombre de Congolais. Les expressions d' "eldorado » ou de «paradis » venant, en amont de l'expérience migratoire, qualifier l'Europe. L'image idyllique de l'Europe a notamment été véhiculée par des musiciens, généralement «Sapeurs » (Gandoulou, 1984) ${ }^{14}$, de passage dans les capitales européennes. Avec les politiques européennes de l'immigration, les Congolais vont en faire une autre expérience ${ }^{15}$. Les appellations de « réfugié » ou « d'immigré » venant récapituler les stigmates statutaires qui se greffent aux différentes étapes du processus de régularisation juridico-administrative, lequel délivre de façon progressive et partielle les droits à l'existence légale sur ces territoires. À cela se greffe un système de discriminations plus ou moins tacite, dans les rapports sociaux, au logement et surtout à l'emploi. Les hommes, dont un grand nombre sont détenteurs d'un ou plusieurs diplômes, y compris de $3^{\mathrm{e}}$ cycle, sont généralement soumis à une très forte déqualification. Pour contourner une perspective professionnelle faite de «petits boulots », certains se reconvertissent alors dans des études d'infirmier, de taximan ou développent des formes d'entreprenariat qui les situent dans l'entre-deux de circulations entre le Congo et l'Europe et de plus en plus vers la Chine, dans la dynamique des nouveaux cosmopolitismes (Tarrius, 2000). Quant à la vie professionnelle des femmes, elle est souvent marquée par des horaires contraignants (soirée, nuit, week-end etc.) et par un cantonnement à des tâches domestiques ou manuelles. Quelles qu'aient été leurs formations antérieures, elles font le plus souvent des ménages. L'ensemble de ces traitements sociaux différenciés, officiels et officieux, participe d'une réalité collective qui se traduit par une nouvelle terminologie. Celle de «parcours du combattant ». À Kinshasa même, alors qu'on pense son projet

14 Le terme de «sape » est à l'origine un sigle, la Société des Ambianceurs et Personnes Élégantes, qui décrit l'apparence et le voyage qui font le Sapeur dans l'entre-deux des allersretours entre Paris et le Congo.

15 En Belgique, les Congolais sont présents plus tôt qu'en France, dès les années 1960. Les liens historiques entre le Congo et la Belgique favorisent la venue d'étudiants, de diplomates, de commerçants et de touristes, qui ne développent toutefois aucune logique d'installation durable. 
migratoire, l'expression qui s'impose est celle de «kobeta libanga », qui signifie littéralement (en lingala) 《taper la pierre » ou «tailler dans le roc », à l'image de l'explorateur anglo-américain Henry Morton Stanley ${ }^{16}$.

Les dispositifs religieux d'accompagnement comme les cellules d'intercession ou les visites à domicile, mais aussi les prières et les cantiques chantés durant le temps de l'adoration, sont autant d'espaces-temps religieux qui rendent perceptibles les situations «d'attente » et de « souffrance » des fidèles, lesquels demandent inlassablement de la «force ». De façon significative, l'item organisateur de la parole pentecôtiste est celui de l'annonce de la délivrance à venir, que les fidèles attendent avec joie et travaillent à mettre en œuvre :

"Toi qui souffres, toi qui te sens humilié, tu dois savoir que Dieu a un plan merveilleux pour toi. Je suis venu t'annoncer que Dieu va agir dans ta vie, que Dieu va faire des miracles, que Dieu va résoudre ton problème, qu'il va te donner des papiers, que tu vas te marier cette année, que tu vas décrocher un travail dans la semaine etc. ».

L'Église se donne alors à voir comme un lieu de culte qui fournit au croyant des ressources pour se penser en dehors des cadres qui régissent une quotidienneté marquée par des assignations identitaires discriminatoires, et dont la pigmentation de la peau est le marqueur majeur. Nous sommes en 2004, lors d'un culte au sein d'une Église implantée en France. Une jeune femme veut témoigner de ce que «Dieu a fait dans sa vie ». Elle s'avance devant l'autel, prend un micro et fait face à l'Assemblée. Dans les Églises de réveil, le témoignage a une fonction de «fortification » et « d'édification » de la foi du Chrétien. Il fait partie intégrante de la mission pour le converti qui doit à son tour témoigner des « grandes choses » que Dieu a faites dans sa vie. Cette jeune femme explique qu'elle recherchait du travail, mais cette fois, " autre chose » que ce qui lui est habituellement proposé [entendre « des ménages »]. Elle vient de décrocher deux stages au sein d'institutions importantes. Et elle nous explique que Dieu a agi dans sa vie de deux manières : d'abord parce qu'elle a "osé » faire des candidatures au sein de ces institutions, et ensuite parce qu'elle a été retenue au Conseil Général et à Airbus, "ce sont des endroits, très, très difficiles à toucher, mais Dieu a fait que j'ai été choisie ». Elle nous explique le parcours qu'elle a effectué : l'Église, la cellule de prières ${ }^{17}$ et puis cette prière improvisée avec des frères et sœurs de l'Église, où elle demande à Dieu de lui donner " de la force » pour l'entretien, que Dieu fasse qu'elle soit «lumineuse » et que ses interlocuteurs «n'entendent pas les bêtises » qui sortiront de sa bouche. L'entretien au Conseil Général est rapide, elle s'inquiète, mais son interlocutrice lui dit qu'elle va personnellement soutenir son dossier, qu'elle a été

16 Alors qu'il entreprenait la construction de la route reliant Matadi et Stanley (1879-1884) pour assurer des échanges commerciaux. Devant tailler à même le Roc, il utilisa de la dynamite et fut surnommé «Bula Matari » (qui en kikongo signifie celui qui taille le roc) par les habitants.

17 Durant la semaine, les Églises ont des cellules d'intercessions et de prière, en général au moins deux fois par semaine, qui s'organisent généralement par quartier, ayant lieu au domicile d'un frère ou d'une sœur de l'Église. 
convaincue. Elle s'engage alors envers son Dieu à témoigner de la gloire de son nom, si elle est reçue. La chose est faite, et quelques jours après, c'est Airbus qui confirmera à son tour l'embauche pour un stage. Elle parviendra qui plus est, à faire différer le deuxième stage pour pouvoir bénéficier de ces deux références qui enrichiront son curriculum vitae. C'est ainsi qu'elle encourage les fidèles à persévérer, "ils n'ont pas vu la couleur de ma peau ou quoi que ce soit, parce que c'est Dieu qui était là ».

Ces stages qui sont objectivés comme des victoires témoignent de la possible mobilité sociale dès lors que le croyant s'en remet à son Dieu. Le modérateur enthousiaste, qui anime le culte ce jour-là, reprendra la parole en disant à ces frères et sœurs :

"Bien-aimés, tout ça c'est pour nous : les institutions, les grandes entreprises... Il faut demander des grandes choses à notre Dieu parce que nous prions un Dieu qui est grand. Si vous priez un Dieu petit, si vous demandez des petites choses, il fait de petites choses. Notre Dieu est un Dieu vivant qui fait de grandes choses, qui fait des miracles, demandez de grandes choses, voyez grand!».

Se « révolutionner » ou « s'évolutionner » sont des néologismes répandus dans ces milieux qui désignent la prise de conscience individuelle, souvent sous forme de vision ou de révélation, de ses propres potentialités et capacités à appréhender le dépassement d'un état d'esprit qui subit, comme l'illustre cet autre témoignage (2005) d'un prédicateur, relatif cette fois à la création d'une Église ${ }^{18}$.

"Quand on est arrivé ici, c'est vrai que beaucoup avaient ce complexe d'infériorité, mais il faut s'évolutionner. Il faut se dire que ce sont des hommes comme nous, voilà. Alors, deuxième chose aussi : ils n'arrivaient pas à prier, complexe de langage aussi, de langue, pardon. Il sait bien parler français, mais pour prier en français, c'est autre chose, alors que les Blancs, heu, pardon, les Français, même si tu parles mal français, je vois personne qui va rigoler de toi, ils rigolent pas. Y'avait ce complexe d'infériorité, si je prie, je vais peut-être mal prononcer, si au lieu de "le" je mets "la", je serai mal vu (...) Personnellement ce qui m'avait encouragé, c'était une dame, elle était espagnole, elle priait avec l'accent espagnol : "mais si cette dame là, qui prie mal, prie, alors pourquoi pas moi ?". Je parle bien français par rapport à elle. Même Pasteur Henri et les autres frères, c'est là où on s'était révolutionnés à prier à l'Église en français (...) Mais comme on était minoritaires à se révolutionner, on a estimé qu'il fallait qu'on fasse une Église, mais qui soit quand même en collaboration avec les Églises de France, pour ne pas être isolés».

Ces récits d'un investissement, par étapes, de la société d'installation insistent sur le processus qui permet d'opérer une «r-évolution » à l'échelle individuelle (et par extension collective). Le droit à négocier sa place (malgré la couleur de sa peau ou malgré les «bêtises » qui pourraient être dites) et à s'exprimer dans la langue de

18 Ce prédicateur fait partie du noyau d'une Église importante dans le sud de la France comptant environ 150 membres. Il importe de préciser que l'équipe dirigeante de cette Église aujourd'hui reconnue, relève d'une origine sociale modeste. 
l'autochtone (malgré les fautes d'orthographe), amène à visibilité un esprit d'entreprenariat, que les candidatures spontanées et l'implantation de l'Église traduisent. On voit par ailleurs, que les étapes, même infimes, de ce qui peut être associé à une avancée dans le processus d'établissement et de mobilité sociale sont énoncées comme des victoires qui rappellent que le succès des entreprises terrestres intervient comme signe de la grâce divine (Weber, 1964).

"On n'est rien, mais avec notre Dieu on peut tout », telle est l'équation résumée de l'affranchissement, annoncé et exhorté, de ces croyants au statut et à l'identité « d'immigré ».

\section{INSTITUTIONNALISATION DE L'ATTENTE ET ESPRIT DE VICTOIRE}

Dans la vie d'une Église peuvent aussi intervenir des évènements qui traduisent la non intervention de Dieu, comme un décès suite à une maladie malgré le témoignage de sa rémission, la réduction à un travail manuel ou domestique, malgré des études supérieures, des papiers qui n’arrivent pas depuis plusieurs années ou encore une expulsion...

Lors d'un culte, dans une Église située à Bruxelles (2006), nous apprenons l'expulsion d'une Sœur, qui était depuis plus d'un mois dans un centre fermé. Certains membres de l'Église accompagnés du Pasteur étaient allés « la visiter ». Durant les cellules d'intercession et les cultes, l'Église avait prié pour que sa situation évolue. Aujourd'hui, le Pasteur annonce cette nouvelle, déjà connue de certains, qui suscite un certain effroi dans l'Assemblée. Ce dernier va répéter son annonce en insistant sur le «Alléluia », auquel doivent répondre les «Amen » approbateurs, qui ne se sont pas encore manifestés. À deux reprises, il exigera cette approbation qui semble rejouer l'adhésion des fidèles, traversés par des sentiments de tristesse et de révolte au regard du « piège » tendu par la police. En effet, suite à une convocation, la Sœur s'était présenté à la police, son avocat lui ayant affirmé que cette étape s'inscrivait dans le processus normal de régularisation de sa situation.

"Bien-aimés, vous savez notre sœur Thérèse était enfermée depuis maintenant un mois et demi. Elle a été renvoyée au pays, Alléluia (Amen), Alléluia (Amen). Bien-aimés, si Dieu a fait que la police prenne notre Sœur et la renvoie au pays, c'est que Dieu a son plan. Dieu pouvait intervenir, s'il ne l'a pas fait, c'est parce qu'il a son plan. Peut- être que notre Sœur va revenir et fera de grandes choses, peut-être que Dieu a besoin d'elle au pays, Alléluia (Amen), Dieu a son plan que l' homme ne peut pas comprendre...».

Cet événement qui pourrait relever d'un contre-témoignage, offre une lisibilité des tensions qui peuvent présider à l'autorité du Pasteur dont le statut d'intermédiaire et d'organisateur terrestre de la délivrance est à la mesure de son pouvoir charismatique et de ses compétences entreprenariales (Dozon, 1995). Mais il nous renseigne surtout sur la direction de cette pastorale chrétienne, résolument optimiste dans la lecture des évènements au point parfois de nier le pouvoir de ces «blocages ». Si «Jésus est la 
solution à tous tes problèmes », la Grâce quant à elle, relève d'un ordre dont la pensée et la temporalité ne sont pas celles de la volonté humaine. Quelle que soit l'apparente injustice des évènements, à l'image de la mise à l'épreuve de Job, se révolter revient à se révolter contre son Dieu. Il importe d'accepter les épreuves, de « s'accrocher ». Le doute étant le meilleur moyen pour ne pas être sauvé. C'est par une foi inébranlable en Jésus et un «travail sur soi », que le croyant pourra voir les miracles et sa vie transformée. Cette posture de «vainqueur », par laquelle le croyant témoigne de la grandeur de son Dieu, se cultive au sein de l'Église de différentes manières : par l'abandon de traditions et des pratiques « païennes », par l'adoption de la morale chrétienne et par l'exercice régulier de pratiques comme la lecture de la Bible, la prière ou le jeûne et bien sûr par une fréquentation assidue de l'Église. Quel que soit le degré de développement de l'Église, le déroulement d'un culte reprend toujours les mêmes séquences temporelles (dont l'ordre peut varier) : «la Louange » (cantiques), « l'Adoration » (prières individuelles et collectives), le prêche, les offrandes et les annonces des activités de la semaine. Les témoignages s'insérant de façon non systématique dans ce programme.

L'annonce de la délivrance à venir (dont la forme ultime est le retour de JésusChrist) structure la majeure partie des prédications. Les problèmes de papiers et d'emploi (dont dépendront l'équilibre d'une famille, d'un couple, les études d'un enfant resté au pays, un mariage ou encore un accès aux soins etc.) sont régulièrement abordés, sous l'angle des situations d'attente qu'ils génèrent, globalement vécus comme humiliantes et déstructurantes. Dans la temporalité du culte, les chants religieux qui mêlent cantiques des Assemblées de Dieu, Gospel et musique des « jeunes » (reggae, rap), sont entrecoupés de temps de prière au cours desquels le modérateur ou le pasteur invitent les fidèles à communiquer directement avec leur Dieu. Chaque voix s'élèvera ${ }^{19}$ laissant libre cours aux émotions du croyant qui peut crier, chanter, pleurer ou encore taper des pieds s'il en ressent le besoin. Dans cette temporalité, l'Église se manifeste comme le lieu d'une mise en scène extraordinaire de « l'Attente ». De l'attente du retour de Jésus-Christ à l'attente du dénouement de situations «bloquées », l'Église offre non seulement un espace d'extériorisation des émotions, mais un lieu de mise en sens des «blocages » qui, quelle que soit leur évolution, s'inscriront toujours dans le «plan merveilleux » de Dieu. C'est dans cette récupération et réinterprétation des situations vécues, transposées dans le registre religieux, que le discours pentecôtiste opère une fonction régulatrice, que je suis tentée de qualifier de forme d'institutionnalisation de l'attente. Ce qui ne doit pas être compris en termes de fixité, mais plutôt en termes d'entre-deux et de pragmatique. La canalisation (discursive et émotionnelle) des sentiments de doute, de révolte ou de désespoir, qui relèvent de la catégorie des mauvais esprits, est une sorte de réorientation qui a à voir avec la dynamique de transformations qu'impulsent les pasteurs. Car, ne l'oublions pas, dans ce parcours du combattant et cette lutte contre les

19 La prière au contraire des cultes qui s'exercent exclusivement en français peut être faite dans une langue «étrangère ». Sachant que la RDC compte quatre langues nationales : le lingala, le kikongo, le swahili et le tshiluba. 
(mauvais) esprits, la figure idéaltypique qui se dégage est celle du Chrétien qui ne s'avoue jamais vaincu. Ce que je vais illustrer par une surprenante réplique d'un Pasteur, résident en Belgique et interrogé dans un cadre non religieux (mariage coutumier), au sujet des politiques de l'immigration qui faisaient l'actualité en France :

"Pour moi on n'a pas à combattre Sarkozy ${ }^{20}$, c'est plutôt un modèle... Il est comme nous, c'est un immigré, mais lui, il est arrivé au top, il faut le prendre comme un exemple».

Cette vue, qui ne pourrait être partagée par l'ensemble des Pasteurs, n'en est pas moins, une rhétorique significative du modèle de réussite qui porte ce discours intégrationniste. La figure du self made man qui se dégage, traduit cet effort que l'individu doit fournir pour rompre avec le statut, souvent qualifié de médiocre, « d'immigré ». C'est à ce niveau qu'une déclinaison toute particulière d'esprits à combattre se dégage, désignant des manifestations émotionnelles et des « constructions » identitaires comme lieux de «blocage »: " je chasse tout esprit de timidité », de peur, de petitesse d'esprit ou encore de médiocrité.

Toujours dans cette «tendance », la lutte contre les stigmates qui «bloquent » ces chrétiens peut passer par une désignation/diabolisation des marqueurs culturels qui en sont à l'origine. C'est alors un référentiel et des catégories justificatrices de rapports sociaux hiérarchisants et asymétriques, qui se dégage. Cela me fut particulièrement perceptible le jour où le Pasteur d'une grande Église située à Bruxelles me demanda (toujours dans un cadre informel), quelle place j'accordais, dans le cadre de ma recherche, à la «paresse » (sic) ? Bien embêtée, je fus obligée d'avouer que la notion de paresse ne m'était jamais venue à l'esprit, mais que je pouvais envisager celles d'attente ou de brutalité d'un système qui pourrait produire des sentiments de « démission » etc. Il acquiesça finalement, considérant qu'au regard de la réalité d'une « immigration non préparée (l'Europe va tout résoudre) » et d'une population «non qualifiée », ou ne pouvant exploiter son capital-diplôme, nonobstant un traitement « parfois raciste », le risque de tomber dans un « assistanat », redoublé de pratiques informelles «illégales » était véritablement «problématique ». Les responsables religieux mettent effectivement en avant le travail effectué auprès des populations « immigrées » et déviantes, en termes « d'utilité sociale », non sans reprendre certains clichés (prostituées, drogués, délinquants etc.). Ce qui ne laisse pas indifférent certains pouvoirs publics qui tendent alors à désigner ces responsables religieux qui prêchent l'excellence, le travail, l'honnêteté et une bonne moralité, comme des responsables communautaires. D'autant que si certaines grandes Églises mettent parfois en place des structures d'accompagnement effectives (mettant à profit les compétences et qualifications de leurs membres : conseil juridique, soutien scolaire, aide alimentaire etc.), il semble que ce soit essentiellement dans le dispositif religieux à proprement parler et les sociabilités qui lui sont associées, que les fidèles se « ressourcent » et « s'édifient».

20 À l'époque où l'actuel Président français, alors Ministre de l'Intérieur, se distinguait par le durcissement de sa politique en termes d'immigration non sans accent xénophobe. 


\section{RELIGIOSITÉ HYBRIDE ET ENTRE-DEUX CULTURELS}

"Dernièrement, le Pasteur Kroll a dit à mon épouse : ton mari n'est plus Africain, il est devenu moderne... tu connais le Pasteur Kroll, quand il dit quelque

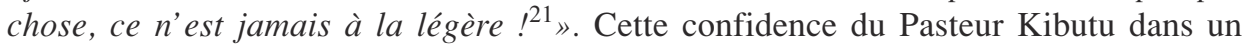
cadre informel est venue clôturer un entretien au cours duquel ce dernier avait développé la question de l'intégration socioprofessionnelle et citoyenne de ses fidèles, qui est au cœur de sa mission. Le Pasteur Kroll lui, est hollandais. Il a, à un moment donné, soutenu le développement de l'Église de l'EIB, l'Église Internationale de Bruxelles $^{22}$, dont le Pasteur Kibutu est le Pasteur principal. Mais il est surtout très actif, depuis des années, dans l'évangélisation au Congo, particulièrement auprès des populations pygmées. Également actif dans l'organisation Campus for Christ ${ }^{23}$ en Europe, il a, lors d'un colloque, rencontré le Pasteur Kibutu qui exerçait dans la même organisation, mais à Kinshasa. Ce partage d'expérience les a rapprochés et de là, est née une collaboration sur le sol européen, concernant notamment le développement de l'EIB, implantée par le Pasteur Kibutu en 1984 à Bruxelles (et aujourd'hui affiliée à la fédération des Églises évangéliques de Belgique). Immergé depuis plus de quinze ans dans les milieux «Blancs », ce dernier considère, en effet, qu'après quatorze années d'études théologiques effectuées en Belgique, son «intégration » au sein du milieu africain relève d'un « choc culturel ». Cherchant à prolonger cette surprenante révélation, je lui demandais en réponse (et à tâtons) si le fait de ne plus être «Africain », d'être « moderne », était de l'ordre d'une « délivrance »:

"Pas du tout, c'est une perte, une perte de mon identité culturelle, mais je la retrouve grâce à l'Église, c'est grâce à l'intégration [dans l'Église] que je peux éviter la césure avec les Congolais (...), ma femme elle, est complètement congolaise, elle n'a pas changé comme moi, elle fait tout congolais, elle mange congolais (...) c'est peut-être parce qu'elle est restée très en contact avec sa mère. ».

Il peut paraître déroutant que le responsable de la seconde plus grande Église congolaise de Bruxelles se situe dans une position d'extériorité culturelle par rapport au groupe religieux à caractère « ethnique » (Fath, 2005) qu'il conduit ${ }^{24}$. Pourtant, le Bishop Martin Mutyebele, Pasteur principal de la «mythique » Nouvelle-Jérusalem, la plus grande Église congolaise à Bruxelles et en Europe ${ }^{25}$ a, lui aussi, évoqué cette

21 Je l'ai connu en assistant aux cours de missiologie que l'EIB prodigue, puisqu'il y animait une partie des enseignements auxquels j'assistais via un autre Pasteur. Je l'ai par la suite interviewé chez lui, en Hollande.

22 Qui compte près de 1000 membres.

23 Organisation internationale qui a pour but d'évangéliser dans les milieux étudiants.

24 Dans l'Assemblée, il y a rarement plus de dix «blancs » lors des cultes. Par contre plusieurs nationalités africaines sont présentes.

25 Comptant plus de trois mille membres, quatre cultes le dimanche (ciblant des publics différenciés socialement et linguistiquement : francophone, lingalaphone et swahiliphone) et vingt-six Églises annexes (en Belgique, en Allemagne, en Russie, en Suisse, en Angleterre et aux États-Unis). 
position d'entre-deux. L'une de ces expressions a eu lieu lors d'une campagne d'évangélisation au théâtre St Pierre, à Bruxelles, en Août 2005. Cette campagne dont l'objectif était d'impulser un mouvement fédérateur auprès des Églises congolaises, fut organisée sur plusieurs jours. Plus de 70 Pasteurs étaient présents. Le Pasteur Mutyebele fut appelé à témoigner. À son arrivée sur scène, le public se leva et l'acclama longuement. Il commença comme il se doit par prier, puis fit le récit des origines de cette « institution » qu'est la Nouvelle-Jérusalem. L'anecdote centrale du récit porta sur la façon dont ses fidèles eurent à négocier l'introduction des rythmes et instruments de musique africains dans l'Église. Lui-même "étant habitué » aux cantiques traditionnels du protestantisme européen et nord-américain qui n'incluaient ni le «tam tam », ni les chants en lingala. Ces leaders religieux, qui se trouvent diriger les deux plus grandes Églises congolaises de Bruxelles font le constat sur un mode plutôt élogieux d'une position d'intermédiaire entre deux univers culturels. La formation puis l'exercice de leurs qualifications au sein d'institutions religieuses établies (et «blanches ») est présentée comme une «intégration » qui aurait pour corollaire une rupture «culturelle », une « césure » avec le groupe d'origine qui deviendrait le lieu de « ré-acculturation » du leader. Cette figure de l'intermédiaire, si elle est effective en regard des structures au sein desquelles certains Pasteurs évoluent et de la mission portée, n'en est pas moins mise en scène comme un marqueur de distinction et d'évolution. Cette forme de présentation de soi, au sens goffmanien du terme, se retrouve dans de nombreuses prédications qui témoignent de la force spirituelle présidant à une intervention divine. Par la restitution de leurs contacts ou des négociations qui ont permis de lever un «blocage » social, les Pasteurs «édifient » la foi des croyants. La démonstration de la subversion des frontières culturelles et/ou raciales qui présideraient aux blocages de «l'immigré » peut concerner l'obtention d'un visa pour aller prêcher aux États-Unis, une négociation avec le propriétaire des locaux pour différer un paiement comme une collaboration avec les réseaux protestants locaux sur des bases « égalitaires » etc. Et c'est en ce sens que l'organisation, de type « ghetto », est dénoncée par les Pasteurs, qui sans toujours y parvenir prêchent contre le « communautarisme ». Ce qui suppose là aussi, des adaptations culturelles et le changement de certaines pratiques, notamment dans la gestion des temporalités et dans l'usage systématique de la langue locale. Il faut «s'ouvrir », « être à l'heure » pour que les «Blancs puissent s'y retrouver ». Et parce que l'accueil est à la mesure de l'intention de l'Église de s'agrandir et surtout de susciter la conversion, l'accueil de nouveaux venus est l'objet d'une attention particulière et d'autant plus s'il est «Blanc ». Ma présence de femme blanche, accessoirement chercheure, a toujours été l'occasion d'un accueil chaleureux (qui en cela ne diffère pas tellement des autres milieux congolais). Par contre, c'est une réelle fierté, dans les milieux religieux que de pouvoir présenter "la sæur Sarah qui prie avec nous ». L'enjeu de ma conversion, et de mon affiliation, s'est d'ailleurs souvent posée, surtout dans les petites Églises, en quête de légitimité, comme si la présence d'un mundele (blanc) dans l'Assemblée, permettait de «décommunautariser » ces Assemblées en prouvant la dimension universaliste de l'appartenance chrétienne. Et a fortiori la capacité de ces chrétiens à « s'intégrer » à l'univers socioculturel de la chercheure cooptée. L'enjeu de cette affiliation visant notamment à me faire passer d'un statut symbolique à celui d'une « connexion » effective (de membre participant activement et financièrement à 
l'avancée de l'œuvre). En situation de «précarité », certains Pasteurs ont ainsi vu en moi une ressource, réelle ou supposée, susceptible d'accélérer l'avancée de « l'œuvre de Dieu », en simplifiant certaines démarches ou en leur donnant une légitimité.

\section{D'UN MODE D'INTÉGRATION À UN AUTRE}

Si certains discours tout en affirmant leur intention de rompre avec un complexe d'infériorité semble l'actualiser, on peut se demander dans quelle mesure des expressions telles qu' "accepter d'évoluer (en étant à l'heure) », "être moderne », "se conduire en civilisé », "sortir de la médiocrité » ou encore par la revendication de certains Pasteurs de "ne pas être un Africain comme les autres », ne seraient pas in fine une forme de rhétorique opportune. Car dans certains contextes, notamment de participation institutionnelle dans les milieux protestants (Demart, 2008b) ou d'évangélisation des autochtones (Demart, 2008a), cette exigence de transformation est double : transformation de Soi et transformation de l'Autre étant indissociables. À ce niveau, le discours religieux élabore alors une critique de l'Europe.

"Nous sommes en Europe, c'est vrai, mais l'Europe a sa mentalité, étant chrétien, nous devons marcher selon ce que la parole de Dieu dit, nous ne devons pas nous conformer à ce monde, nous sommes un peuple qui est mis à part ».

Cette référence à la «mentalité » de l'Europe désigne la perte des valeurs chrétiennes et de la foi, l'individualisme, la peur de l'altérité et « l'accueil » qui en découle, l'indifférence dans les rapports de voisinage, le divorce généralisé, la difficulté à éduquer ses enfants en Europe face à une autorité parentale défaillante et généralisée ou encore la nudité banalisée de la femme dans l'espace public. Si ces critiques sont globalement transversales aux populations congolaises, elles sont, dans le cadre religieux, appréhendées sous l'angle de la déchristianisation de sociétés, que le confort matériel a éloigné de Dieu. Au travers du récit d'un peuple élu et persécuté, marchant vers la terre promise ou attendant le retour du Christ, la Bible fournit une caution et une arme symbolique pour des collectifs de migrants qui expérimentent l'indésirabilité et la non-valeur de leur statut d'immigré-es « non choisis ». L'affirmation d'une seule et unique frontière, celle qui sépare les chrétiens des païens, les convertis des non-convertis, annule alors ipso facto le bien fondé des autres frontières qu'elles soient ethniques, nationales, culturelles ou raciales. Par son alliance avec Dieu, qui devient un partenaire permanent des entreprises terrestres, le converti peut envisager sinon un renversement de l'ordre des hiérarchies sociales, du moins sa légitime traversée. En cela, l'identité chrétienne fournit une garantie au croyant : celle de sortir à plus ou moins court terme, vainqueur de ce «parcours du combattant». Dès lors, bien sûr, qu'il en prend la décision de façon indéfectible. Cette posture sous-tend une critique à l'endroit d'une Europe qui a non seulement failli à ses idéaux démocratiques, mais trahi les valeurs de l'Évangile, qu'elle avait pourtant elle-même « apporté aux Africains ». Dans une vision comparative avec le modèle sociétal américain, l'Europe, catholique, et de surcroît déchristianisée apparaît d'autant plus fermée à l'altérité : "Aux États-Unis, on prend le meilleur, ici, on ne vous prend pas parce que vous êtes différents », récapitule ce Pasteur qui prêche régulièrement dans des églises européennes et nord-américaines (et qui prépare, par ailleurs, dans une 
université américaine un doctorat sur les stratégies de pénétration de l'Évangile en milieu européen). La plus forte représentativité socioprofessionnelle des « Noirs » aux États-Unis, à laquelle les « jeunes » sont tout particulièrement sensibles, conforte cette idée d'avancement. De plus, des grandes croisades comme celles d'Osborn ou de Pat Robertson, les passages télévisés sur la chaîne congolaise de Billy Graham dans les années 1980 ou des organismes comme le Full Gospel Businessmen Fellowship ou Campus for Christ, ont durablement marqué l'histoire du Réveil congolais. Enfin, si la plupart des Églises se développent sur un mode d'autofinancement, il n'échappe à personne que la Nouvelle Jérusalem doit son développement remarquable en partie aux financements des Églises américaines de la Church of God, dont le Pasteur Mutyebele est aujourd'hui le représentant en Belgique.

Lorsque les Pasteurs rappellent que "nous prions un Dieu "vivant" et non "mort" comme chez les catholiques ", ils font appel à une foi sans borne et à un Dieu qui, depuis les temps apostoliques, "n'a pas changé ». Mais la référence au Dieu «mort» des catholiques est aussi une référence à une histoire plus lointaine, plus ou moins théorisée selon les Pasteurs. Dans l'histoire du Congo, le catholicisme a, en effet, été un instrument majeur de l'État colonial ${ }^{26}$. L'objectif qui fut énoncé sans ambages aux missionnaires en partance pour le Congo, dans une lettre mémorable du roi Léopold II, était celui de la préparation psychologique des colonisés à se soumettre à la grande entreprise de domestication et d'exploitation économique du Congo ${ }^{27}$. Les « croisades » et évangélisations des années 1970-1980 qui venaient révéler le caractère accessible du Salut et les miracles sans limite d'un Dieu «vivant », qui n'a pas changé (« contrairement à ce qui nous avait été dit ») renversaient un ordre de centralité instauré pendant la période coloniale (1885-1960). Cette (ré)appropriation des voies et modes d'accès du Salut, s'est accompagnée d'une reprise et d'une intensification de la lutte contre les esprits telle que, dans certains cas, la «ligne de partage » (Tonda, 2002) entre univers biblique d'une part, et univers sorcellaire, magique ou traditionnel, d'autre part, n'est pas identifiable. Au point que certains anthropologues y voient une forme de pentecôtisme autre, qualifiant cette nouvelle vague, de " néopentecôtiste » (Laurent, 1999 ; Corten, 1999). La tension entre, continuité et rupture, intelligible dans l'articulation « chasse aux démons », « diabolisation des traditions » et « Évangile de la prospérité » (Marshall-Fratani, 2001), pose selon moi, avant tout la question du cadre spatio-temporel susceptible de restituer cette forme religieuse. Un bref détour vers les rues de Matonge (le quartier congolais à Bruxelles), de Château Rouge (Paris) ou encore vers les vitrines de nombreux commerces congolais, nous donnera un élément de réponse ou plutôt de questionnement. On peut y voir, en effet, de façon très

26 Même si, par la suite, durant le pouvoir dictatorial de Mobutu, le catholicisme a été un contre pouvoir, se donnant à voir comme religion d'opposition dans un contexte toutefois où certains « intérêts » étaient menacés, à l'image de la politique de l'authenticité (en 1972) qui rebaptisa le Congo, Zaïre et exigea de tous les « citoyens »d'abandonner leurs noms chrétiens pour des noms locaux.

27 Lettre du roi Léopold II adressée aux missionnaires et reprise par le Ministre des colonies M. Jules Renquin en 1920 avec les premiers missionnaires catholiques du Congo belge, cf. Avenir colonial Belge, 30 octobre 1921, Bruxelles. 
régulière, des affiches annonçant la venue d'invités exceptionnels, d'Allemagne, de Grande-Bretagne, du Congo ou encore des États-Unis (Congolais en migration ou nationaux). Pour des séminaires de quelques jours ou pour une conférence, ces prédicateurs développeront le thème du leadership : «Vivre le leadership », l' «Impact d'un leadership spirituel », le leadership comme stratégie d'évangélisation, comme gestion de la vie professionnelle et sociale ou encore comme stratégie de management pour l'Église etc. Plus souvent recherchés par les Hommes de Dieu, mais pas uniquement, ces enseignements mobilisent un concept évocateur de réussite, d'excellence et d'ouverture sur l'international. Même si cette internationalité peut se révéler relative, le leadership occupe une place de choix dans le panel des "techniques de soi » de cette offre religieuse. Il apparaît in fine comme un aboutissement de la compétence entreprenariale et charismatique à développer en soi pour accéder aux promesses de la mondialisation

\section{DE L'INJONCTION CONTRADICTOIRE À L' INTÉGRATION}

En 2006, Lydia Mutyebele, fille du Pasteur Martin Mutyebele de la Nouvelle Jérusalem, active dans l'Église de son père (où elle dirige notamment une chorale de gospel) et avocate de formation entrait sur la scène politique belge en se présentant aux élections communales. Candidate $\mathrm{CDH}^{28}$ pour la ville de Bruxelles puis au Sénat (2008), la percée de cette nouvelle candidate ne laissa pas indifférent. Sur sa carte de visite, laissée à disposition dans plusieurs lieux publics, notamment dans les ngandas ${ }^{29}$ de Matonge (quartier congolais à Bruxelles), on pouvait prendre connaissance de son combat politique et citoyen. Lequel appelait à lutter " contre l'indifférence, l' exclusion et la médiocrité ». De façon inédite et récapitulée, s'énonçait un appel à des transformations sociétales qui mettait au même niveau l'indifférence, l'exclusion et la médiocrité. Si l'indifférence et l'exclusion venaient traduire des formes de discriminations entendues comme freins à « l'intégration », la convocation dans l'espace public de la lutte contre la médiocrité visait elle, à n'en pas douter, la « communauté congolaise ». On retrouve là un thème qui circule dans les milieux du Réveil, en France comme en Belgique. Toutefois, si la «médiocrité »vient effectivement qualifier le statut de "l'immigré », les conditions de la rupture avec cette identité stigmatisante relèvent par contre, de logiques différenciées. Dans ce contexte électoral précis par exemple, la référence à cette « tare » est non seulement l'indicateur d'un vote ethnique $^{30}$, mais la référence à un modèle méritocratique qui invite à prendre en charge ou à résoudre, de façon individuelle la question du stigmate. Toutefois, dans d'autres

28 Le parti Centre Démocrate Humaniste. Lydia Mutyebele, était la $25^{\text {ème }}$ candidate sur la liste $\mathrm{CDH}$ pour la circonscription de Bruxelles-Ville comprenant Bruxelles, Laeken, Neder-OverHeembeek, Haren qui est l'une des 19 communes de Bruxelles.

29 Les ngandas sont des bars-restaurants en général stigmatisants dans ces milieux religieux car associés à la bière, aux femmes libres, à la débauche et aux pêchés.

30 Malgré le déni de la direction du $\mathrm{CDH}$, le parti fut traversé par de fortes controverses au sujet de ce vote communautaire. Les scores de Bertin Mampaka, de Lydia Mutyebele ou encore de Nestorine Kimbondja Kalengi, relevant effectivement de stratégies ciblées cf. «le stemblock à la manière $\mathrm{CDH}$ », le Soir, 13 octobre 2006. 
contextes, le refus de cette «médiocrité » passe par une «politique » qui peut concerner les bâtiments, l'organisation des églises ou encore tout un ensemble de négociations avec les protestantismes locaux. La "médiocrité » étant alors une construction sociale (situations d'irrégularité, de précarité, préjugés, misérabilisme etc.) avec laquelle il « faut» rompre. Que ce soit à l'échelle de l'individu ou du groupe, l'esprit d'entreprenariat qui se dégage de ce discours religieux affirme un droit et une intention : ceux de rompre avec la figure stigmatisée et stigmatisante de «l'immigré » qui situe ces collectifs en marge des circuits classiques de l'intégration et de l'insertion sociale. Par ailleurs, c'est à partir de la perte des valeurs morales ou de la non application des valeurs chrétiennes dans les sociétés européennes, que ces collectifs émettent une critique vis-à-vis de cette Europe qu'ils entendent conquérir en négociant leur droit à s'insérer dans l'ordre des hiérarchies et des légitimités autochtones. La référence au modèle américain que l'on peut dégager comme "forme ultime de la civilisation de Dieu » (Tonda, 2000) semble avant tout être le lieu de l'expression d'un refus. Rappelant, si besoin était, que "la décolonisation n'a pas mis un point final à la question de savoir que faire des histoires partagées une fois que celles-ci ont été plus ou moins désavouées " (Mbembe, 2005 : 141). Car dans la structure même de son discours (et dans la grande variabilité de ses formes), le pentecôtisme congolais élabore un système de pensée et de croyances qui repose sur une bipolarité hiérarchisante (esprits/Esprit-Saint, Bible/Sorcellerie etc.) forgée par l'évangélisation coloniale (Tonda, 2000). Plus efficace et plus puissant, le pouvoir biblique l'est donc aussi en termes de légitimité, puisque c'est du point de vue des valeurs chrétiennes, que ces Pasteurs posent la question de l'universalisme, pour contredire le bien fondé de certaines stratifications sociales et leur durabilité. À l'intersection de deux modèles, celui d'une intégration pensée comme assimilation et celui d'un communautarisme, ou d'une ethnicité, perçu comme enfermant, ces collectifs définissent leur « combat pour l'intégration ». L'accent porté sur l'individu reprend une idée forte dans les nouveaux mouvements religieux, selon laquelle "le changement de soi est une manière de changer la société » (Marshall-Fratani et Péclard, 2002). L'impératif d'excellence et de bonne moralité versus la «médiocrité » étant tributaire de l'idée de la toute potentialité de l'individu, par l'alliance avec le divin versus de l'incapacité individuelle à se « r-évolutionner » en rompant « en soi » avec ce qui se manifeste comme un blocage « social ». Cette dynamique de transformations amène à visibilité deux logiques, que l'on peut schématiquement identifier comme de continuité et de rupture. La référence américaine, à travers le réveil religieux et le modèle du self made man soutient la rupture avec les multiples figures de l'altérité construites dans les interactions passées et présentes avec l'Europe : le colonisé, le païen, l'évangélisé, l'étranger et l'immigré. On pourrait, d'ailleurs, se demander si les transformations suggérées à l'échelle de l'individu, en termes de bonne moralité et de distinction d'une part et l'assimilation de « tares », comme la paresse ou la médiocrité, à des attributs collectifs, d'autre part, n'actualiseraient pas des figures coloniales qu'il s'agirait alors de restituer en fonction de leurs usages, contextes et ancrages dans des formes collectives de revendications afin de dégager les différentes stratégies élaborées à partir de ces constructions identitaires. En tout état de cause, si l'on peut s'accorder sur la définition donnée par Bordes-Benayoun et Schnapper (ibid.) de l'intégration comme reconstruction $a$ posteriori et mise en perspective d'un processus interactif entre société d'installation 
(ou de transit) et les migrants, alors on doit considérer que ces recompositions culturelles et identitaires sont de toutes façons, des indicateurs d'un rapport qui déborde le seul cadre des interactions quotidiennes ou de la culture du groupe. Ce qui, dans notre contexte, supposerait de pouvoir restituer le continuum entre contexte colonial et contexte post-colonial de la migration, que ce discours religieux sur l'intégration amène à visibilité. En effet, dans son caractère hybride, évolutif et malléable, cette religiosité s'inscrit dans une logique de continuité qui convoque l'histoire. Sans qu'elle ne fasse l'objet à proprement parler de ce discours religieux, on peut repérer une sorte d'inversion des temporalités, laquelle nous renseigne sur le rapport macrosocial, culturel et historique qu'actualise cette expérience migratoire collective. L'espace-temps du culte (et non de l'Église) qui, on l'a vu, institutionnalise une attente constitutive de ces "parcours de combattants », offre une lisibilité de cette inversion. Car, ce n'est pas ou plus, l'histoire passée qui permet d'éclairer un présent conflictuel, «bloqué » ou malheureux, mais bien l'avenir « délivré », victorieux et maîtrisé par le chrétien et décrit dans les Écritures qui est organisateur du présent. Dans cette inversion des temporalités, la tension entre continuité et rupture semble trouver une issue pragmatique qui a véritablement fonction de régulation. Et c'est bien, dans l'imbrication des référentiels temporels, culturels, sociétaux ou encore territoriaux, que mobilise ce discours pro-intégration, que l'on peut saisir la façon dont ces collectifs expérimentent l'intégration ou plus exactement la capacité d'intégration de sociétés qui, elles aussi, malgré tout, sont issues de l'histoire coloniale (Blanchard, Bancel et Lemaire, 2006). La construction d'une ethnicité, y compris dans le cas de l'exception française manifestant un « décalage » entre discours et pratiques institutionnels (CostaLascoux, 2006), se donnant ici à voir, comme un marqueur significatif de l'injonction contradictoire à l'intégration à laquelle se confrontent ces collectifs. 


\section{Références bibliographiques}

BLANCHARD Pascal, BANCEL Nicolas et LEMAIRE Sandrine (2006) La fracture coloniale, éd. La découverte, $311 \mathrm{p}$.

BORDES-BENAYOUN Chantal, SCHNAPPER Dominique (2007) Diasporas et nations, éd. Odile Jacob, 222 p.

CORTEN André, MARY André (2000) Imaginaires politiques et pentecôtismes, Afriquel Amérique Latine, éd. Karthala, 368 p.

CORTEN André (2001) Instruments de l'Impérialisme ou culture populaire ? Explosion des pentecôtismes africains et latino-américains, le Monde diplomatique, Décembre 2001.

COSTA-LASCOUX Jacqueline (2006) L'intégration à la française : une philosophie à l'épreuve des réalités, Revue Européenne des Migrations Internationales, volume 22, n 2, pp. 105 125 .

DEMART Sarah (2008a) «La recomposition de l'ordre des hiérarchies par le pentecôtisme congolais (République démocratique du Congo) à travers l'évangélisation des autochtones (France et Belgique) » in Migration et Religion en France 1 (XIX ${ }^{e}-X X^{e}$ siècles), les Cahiers de la Méditerranée, $\mathrm{n}^{\circ} 76$, Juillet 2008.

DEMART Sarah (2008b) «De la "secte" au représentant communautaire... Processus de reconnaissance différenciés des églises de réveil congolaises (RDC) dans les contextes migratoires français et belges » in Les nouvelles minorités religieuses dans l'espace public : Demandes de reconnaissance et réponses institutionnelles dans les pays occidentaux, éd. la Découverte, septembre 2008.

DEVISCH René (1996) "Pillaging Jesus": Healing churches and the villagisation of Kinshasa, Africa, 66 (4), pp. 555-585.

DOZON Jean-Pierre (1995) La cause des prophètes, Paris, Le Seuil, 299 p.

FANCELLO Sandra (2006) Les aventuriers du pentecôtisme ghanéen. Nation, conversion et délivrance en Afrique de l'Ouest, Karthala, 378 p.

FATH Sébastien (2005) Du ghetto au réseau, le protestantisme évangélique en France (18002005), Labor et Fides, 425 p.

FOURCHARD Laurent, MARY André et OTAYEK René (2005) Entreprises religieuses transnationales en Afrique de l'ouest, Karthala, 538 p.

GANDOUlOU Justin Daniel (1984) Entre Paris et Bas-Congo, éd. Centre Georges Pompidou, $213 \mathrm{p}$.

GOFFMAN Erving (1973) Les mises en scène de la vie quotidienne, les éditions de minuit, $256 \mathrm{p}$.

LAURENT Pierre Joseph (2003) Les pentecôtistes du Burkina Faso, mariage, pouvoir et guérison, éd. Karthala, 442 p.

LEVITT Peggy (2004) Redefining the boundaries of belonging: The institutional character of transnational religious life, Sociology of religion, vol. $65, \mathrm{n}^{\circ} 1,2004$, pp. 1-18.

MARSHALL-FRATANI Ruth (2001) Prospérité miraculeuse : Pasteurs pentecôtistes et argent de Dieu au Nigeria, Politique africaine, ${ }^{\circ}$ 82, Karthala, Paris pp. 24-44.

MARSHALL-FRATANI Ruth, PECLARD Didier (2002) «La Religion du sujet en Afrique » in les Sujets de dieu, politique africaine, $\mathrm{n}^{\circ} 82$, pp. 5-19.

MBEMBE Achille (2005) La république et « l'impensé de la race in Blanchard P., Bancel N. et Lemaire S., la fracture coloniale, éd. La découverte, pp. 139-141.

TARRIUS Alain (2000) Les nouveaux cosmopolitismes, éd. De l'Aube, 265 p.

TONDA Joseph (2000) « Capital sorcier et travail de Dieu », Politique africaine, pp. 48-65.

TONDA Joseph (2002) La guérison divine en Afrique centrale (Congo, Gabon), éd. Karthala, $243 \mathrm{p}$.

WEBER Max (1964) l'éthique protestante et l'esprit du capitalisme, Plon, 285 p. 


\section{Le « combat pour l'intégration » des églises issues du Réveil congolais (RDC)}

\section{Sarah DEMART}

Cette contribution se propose de mettre en perspectives un discours sur « l'intégration » qui se développe depuis quelques années, dans des églises à caractère " ethnique » (France-Belgique). D'obédience pentecôtiste et évangélique, ces églises issues des migrations congolaises (RDC) se comptent à l'échelle de l'Europe, par centaines, pour ne pas dire par quelques milliers. Si cette nouvelle branche du protestantisme donne à voir un mouvement pluriel et fragmenté, des constantes sont toutefois observables, notamment l'affirmation du droit à se penser en dehors de logiques d'assignation à l'altérité. Il en résulte un discours intégrationniste inédit qui se donne à voir comme rupture avec un rapport ancien qu'actualiserait l'ordre des hiérarchies et des centralités autochtones.

\section{The "Fight for Integration" of the Congolese Revival (DRC) Churches}

\section{Sarah DEMART}

This article proposes to analyse the discourses on "integration" developed during the last few years within various "ethnics" churches (France-Belgium). Of Pentecostal and Evangelical obedience, these churches, stemming from Congolese's migrations (DRC), can be estimated to several hundreds and even up to a few thousands in Europe. Some constant can be observed in this new branch of Protestantism despite its fragmented and plural movement, particularly the right to think oneself beyond the logics of reduction to otherness. The integrationist discourse produced constitutes a real break with the ideas carried by the autochthon order of both hierarchies and centralities.

\section{La "lucha para la integració" de las iglesias nacidas del Renacimiento congolès (RDC)}

\section{Sarah DEMART}

Esta contribución propone poner en perspectivas un discurso de "la integración" que se desarrolla desde hace algunos años en iglesias a carácter "étnico" (Francia-Bélgica). De tendencia pentecostal y evangélica, estas iglesias nacidas de la migración congolesa (RDC), se cuentan en Europa por centenas, sino algunos millares. Esta nueva rama del protestantismo muestra un movimiento plural y fragmentado, sin embargo las constantes son siempre observables, particularmente la afirmación del derecho a pensarse fuera de lógicas de asignación a la alteridad. De esto resulta un discurso integracionista inédito que se muestra como la ruptura a un viejo lazo que actualiza el orden de las jerarquías y de las centralidades autóctonas. 


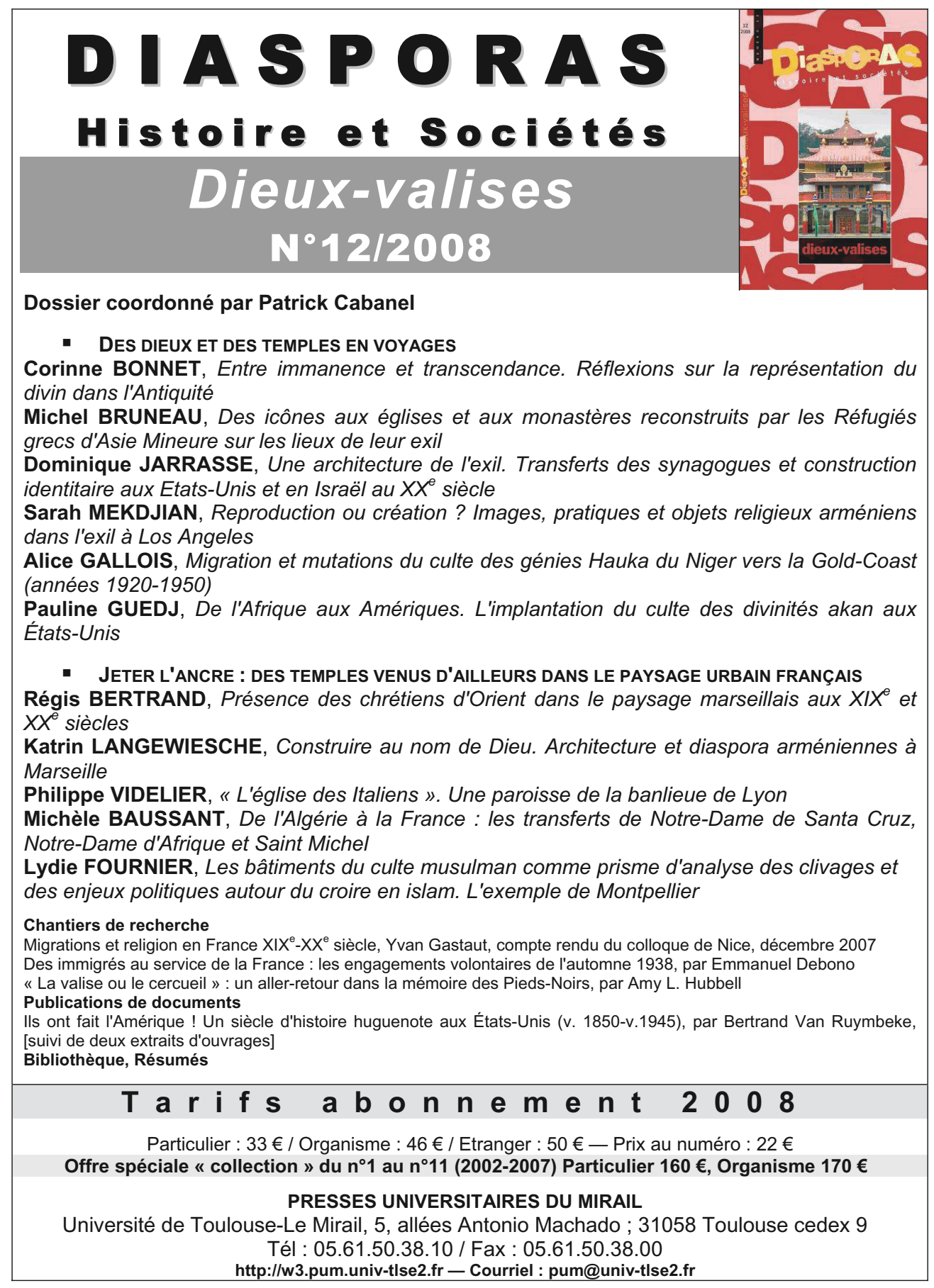

